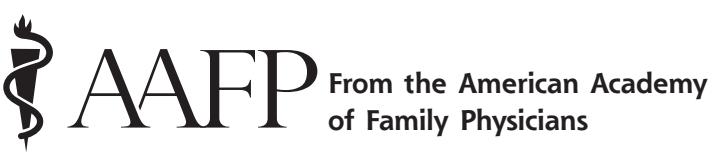

Ann Fam Med 2015;13:186-187. doi: 10.1370/afm.1571

\section{NEW GUIDELINE COVERS LABOR, VAGINAL BIRTH AFTER CESAREAN}

Cesarean deliveries account for 1 in every 3 births in the United States. So when a mother who previously had a cesarean procedure asks a family physician what options she has for another birth, it's important that she knows what she can expect from both another cesarean and a vaginal birth after cesarean (VBAC).

To better inform the discussion of and planning for labor after cesarean (LAC) and VBAC, the AAFP has created an evidence-based, peer-reviewed guideline, http://www.aafp.org/patient-care/clinicalrecommendations/all/vaginal-birth-after-cesarean.html, for family physicians that lays out short- and long-term health outcomes associated with the process.

Guideline panelist and family physician Valerie King, MD, MPH, of Portland, Oregon, said these patients need to know LAC is safe and appropriate.

"We need to counsel women so that they know the risks and benefits, and we need to actively work to make sure they have the option of LAC, if that is what they choose, even if that means we need to send them on to another facility," King said.

\section{Guideline Overview}

To create this update of its 2005 guidance document, the AAFP's Commission on Health of the Public and Science appointed a guideline development group that included family physicians and representatives from the American Congress of Obstetrics and Gynecologists (ACOG), the American College of NurseMidwives, and Childbirth Connection, a nonprofit organization focused on maternity care.

The primary literature source for this update was a 2010 evidence report (http://www.ahrq.gov/research/ findings/evidence-based-reports/vbacup-evidencereport.pdf) on VBAC produced by the Oregon Evidence-based Practice Center for the Agency for Healthcare Research and Quality (AHRQ). A search of the literature published on this topic since that 2010 report also yielded 2,932 articles, of which 19 were deemed appropriate for the evidence review.

The guideline update was warranted, said the document's authors, because of the "documented decline in the rate of VBAC and increase in the rate of cesarean deliveries and accompanying maternal morbidity."
A slowdown in the popularity of LAC/VBAC began in 1996, when a study (http://www.nejm.org/doi/ full/10.1056/NEJM199609053351001) published in the New England Journal of Medicine raised concerns that LAC increased the likelihood of uterine rupture. Then in 1999, ACOG published a practice bulletin, revised in 2010, (http://www.acog.org/Resources_And_Publications/ Practice_Bulletins/Committee_on_Practice_Bulletins_ Obstetrics/Vaginal_Birth_After_Previous_Cesarean_Delivery) that established a standard of immediate physician availability to provide care and emergency delivery during LAC. The combination of these releases sparked controversy and a discussion about the appropriateness of LAC, which led to a decrease in the number of VBACs soon thereafter, the guideline stated.

By 2009, cesarean birth rates had reached an all-time high of almost $33 \%$ and have since hovered at that level.

The 2010 AHRQ evidence report concluded that LAC actually was a safe choice for the majority of women who had a prior cesarean delivery. It also found emerging evidence that multiple cesareans could lead to serious maternal harm. These findings, along with other more recent supportive resources, led the Academy to update its guideline.

\section{Need-to-Know Guideline Points}

According to King, it's important that family physicians ensure a woman who previously has had both vaginal and cesarean births has the opportunity to pursue LAC. "These women actually have a much lower chance of needing another cesarean than a normal first-time mother," she said.

Regarding who shouldn't attempt LAC, King said the process is not recommended for those with a previous vertical uterine incision or vertical extension of a transverse incision, or for those who have had transmural uterine surgery.

The AAFP recommends induction of labor (IOL) for women with a previous cesarean who have a medical indication for induction of labor and are planning to pursue LAC/VBAC.

King outlined the recommended induction process for a woman planning LAC/VBAC:

- Make sure a medical indication for IOL exists. "If a woman just happens to be at 41 weeks of gestation and has no medical indication for IOL, then we would recommend waiting rather than routinely asking her to have an induction," she said

- When possible, use a mechanical means of cervical preparation (such as a cervical dilator balloon) if cervical preparation is required for IOL

- Misoprostol should never be used for cervical preparation in the third trimester for women with a prior cesarean birth 
- When the Bishop score is reasonable (>6-8), IOL can proceed using an infusion of oxytocin

\section{Weighing Benefits, Harms}

The guideline suggests family physicians discuss with every patient who has had a previous cesarean the short- and long-term benefits and harms of planned LAC/VBAC for both mother and infant.

In the short term, said King, nearly all maternal outcomes are equivalent or better with LAC vs another cesarean: fewer maternal deaths, fewer hysterectomies, less blood loss, and fewer fevers.

"However, women who have had a prior cesarean birth are at a higher risk of uterine rupture during labor (about 3 cases of uterine rupture in 1,000 labors), and uterine rupture can increase the risk to the fetus and infant," she said.

On average, there are about 130 fetal/neonatal deaths per 100,000 births with LAC compared with about 50 per 100,000 with repeat cesarean birth, according to the guideline. "The risk to the fetus is highest when there is a uterine rupture, so counseling and care need to carefully address the risks of uterine rupture for each woman," King said, adding that delivering another infant within 18 months of having had a cesarean birth increases the risk of uterine rupture. "However, most women can very safely have LAC in a facility where rapid cesarean delivery can occur in the event of uterine rupture," she noted.

As to long-term benefits and harms, King said that for women planning future pregnancies, each cesarean birth increases the risk of placenta previa and other placental complications.

For example, women with 1 previous cesarean birth face a risk of hysterectomy of about 400 per 100,000 pregnancies, and women with 5 previous cesareans have a hysterectomy risk of about 9,000 in 100,000 pregnancies, she said.

"There really aren't enough data to assess the longterm risk to the fetus/newborn, but there is currently a lot of ongoing research about potential lifetime risks of being born by cesarean-so stay tuned," King said.

Not every family physician handles labor and delivery for their patients, King acknowledged, but every one of them has a role in this process.

"Family physicians are at the front lines for good reproductive health care," she said. "Even if they don't provide maternity care, they should be asking women about their childbearing plans, providing appropriate contraceptive services and encouraging women with a prior cesarean birth to wait a minimum of 18 months before having another baby."

Chris Crawford AAFP News

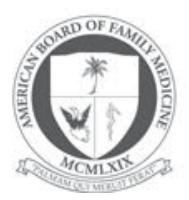

From the American

Board of Family Medicine

Ann Fam Med 2015;13:187-188. doi: 10.1370/afm.1773

\section{KENDALL M. CAMPBELL, MD SELECTED AS 2014 PUFFER/IOM FELLOW}

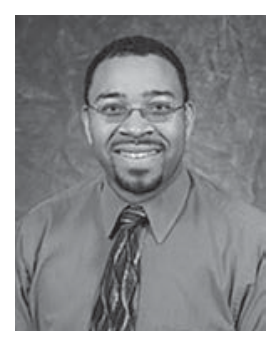

The Institute of Medicine (IOM) has selected Kendall M. Campbell, MD, as the 2014 James C. Puffer, MD/American Board of Family Medicine Fellow. Dr Campbell is an associate professor in the department of family medicine and rural health and co-director for the Center for Underrepresented Minorities in Academic Medicine, Florida State University, Tallahassee.

Dr Campbell is from the rural community of DeFuniak Springs, Florida, and was one of the first Science Students Together Reaching Instructional Diversity and Excellence (SSTRIDE) mentors and instructors. SSTRIDE seeks to identify and encourage middle and high school students from underrepresented backgrounds, including those from rural communities. He became a part of the program in 1994 while an undergraduate student and chemistry pre-med major at Florida A \& M University. After completing his undergraduate degree, Dr Campbell attended the Program in Medical Sciences, a 1-year program created to help promote diversity in Florida's medical schools by attracting more students from backgrounds underrepresented in medicine and in the Florida physician workforce. After the completion of medical school, he went on to become a board-certified family physician with special interests in underserved care and teaching. Dr Campbell is the learning center advisor for the Bridge to Clinical Medicine master's degree program, a program to increase underrepresented groups in medicine, and as co-director of the Center for Underrepresented Minorities in Academic Medicine, conducts research to study issues affecting underrepresented minority faculty in medical education. He sees patients at Bond Community Health Center, a community health center for the underserved.

As a Puffer/ABFM/IOM Anniversary Fellow, Kendall will receive a research stipend of $\$ 25,000$. Named in honor of James C. Puffer, MD, president and chief executive officer of the ABFM, the fellowship program enables talented, early-career health policy and sci- 